\title{
MODELOS DE AJUSTE PARA CURVAS DE LACTACIÓN DE VACAS EN CRIANZA INTENSIVA EN LA CUENCA DE LIMA
}

\author{
Luis Rodríguez Z. ${ }^{1}$, Miguel Ara G. ${ }^{2}$, Héctor Huamán U. ${ }^{3}$ y Luisa Echevarría C. ${ }^{4}$
}

\section{Abstract}

The description of lactation curves by mathematical models could be an effective tool for management decisions in dairy cattle production systems. In this study the comparative fit of three models: negative exponential $\left(i=\left(\hat{a}_{0} e^{-\hat{a} l x}\right)\right.$, incomplete gamma $\left(i=\hat{a}_{0} x^{\hat{a} l e-\hat{a} 2 x}\right)$ and fifth order polynomial $\left(i=\hat{a}_{0}+\hat{a}_{1} x+\ldots+\hat{a}_{5} x^{5}\right)$ was evaluated both between and within lactations using 8,763 milk yield records from 770 lactations for the first two models, and 7,228 records from 647 lactations for the third model. Milk yield records included the period 1997-2001 and were collected from five dairy farms located in Lima. The models were compared on the basis of the adjusted multiple coefficient of determination $\left(R_{a j}^{2}\right)$, the residual standard errors $(E E R)$, the standard errors of the predicted yield on day $305\left(E E_{305}\right)$, and the residual scatter plots. Additionally, the effect of parity, calving season and the 1998 El Niño phenomenon (FEN) on the basic and derived parameters of the models were estimated using general lineal models. In terms of fitness, the models ranked fifth order polynomial > incomplete gamma > negative exponential both between and within lactations. However, the fifth order polynomial model had several shortcomings like higher $E E_{305}$, a tendency of overestimate late lactation yields, and a requirement of a minimum number of records. These left incomplete gamma as the model of choice, being capable to explain $72 \%$ of the yield variation within lactations and having an $E E_{305}$ of only $3.3 \mathrm{~kg}$. Parity and calving season affected significantly most of the basic and derived parameters of the models, whereas the FEN only affected the $\beta$ parameter of the exponential negative and the peak yield predicted by the incomplete gamma model. All of the three mentioned factors had significant effect on the total yield predicted for a 305 day-lactation.

Key words: lactation curve, mathematical model, dairy cow, milk yield

\section{Resumen}

El uso de modelos matemáticos para describir curvas de lactación suministra valiosa información para la toma de decisiones en sistemas de producción de leche. En este estudio se evaluó comparativamente el ajuste de los modelos exponencial negativo $\left(i=\hat{a}_{0} e^{-\hat{a} l x}\right)$, gamma incompleto $\left(i=\hat{a}_{0} x^{\hat{a} l e-\hat{a} 2 x}\right)$ y polinomial quinto $\left(i=\left(\hat{a}_{0}+\hat{a}_{1} x+\ldots+\hat{a}_{5} x^{5}\right)\right.$ entre y dentro de lactaciones, y se estimó el efecto del número de lactaciones, estación de

\footnotetext{
${ }^{1}$ Universidad Nacional San Cristóbal de Huamanga. E-mail: larzvet@yahoo.com

${ }^{2}$ Centro de Investigaciones IVITA, FMV-UNMSM

${ }^{3}$ Laboratorio de Bioquímica, Nutrición y Alimentación, FMV-UNMSM

${ }^{4}$ Facultad de Veterinaria y Zootecnia, Universidad Peruana Cayetano Heredia
} 
parto y fenómeno El Niño 1998 (FEN) sobre los parámetros básicos y derivados de los modelos. Se usaron 8,763 registros de producción correspondientes a 770 lactaciones, provenientes de 5 establos de Lima, entre los años 1997-2001. La bondad comparativa de ajuste de los modelos se evaluó en base al coeficiente de determinación múltiple ajustado $\left(R_{a}^{2}\right)$, al error estándar residual $(E E R)$, al error de predicción de la producción en el día 305 $\left(E E_{\text {sos }}\right)$ y al examen de la dispersión de residuos. El efecto de los factores no genéticos se estimó mediante ANVA. La bondad de ajuste según $R_{a j}^{2}$ tuvo el siguiente orden: polinomial quinto $>$ gamma incompleto > exponencial negativo, tanto entre lactaciones como dentro de lactaciones. Sin embargo, el modelo polinomial mostró un $E E_{\text {sos }}$ relativamente alto, una tendencia a sobreestimar rendimientos en la lactación tardia y un requerimiento comparativamente alto en el número de registros por lactación, lo cual deja como opción recomendable al modelo gamma incompleto. Este modelo explicó e1 $72 \%$ de la variación en producción durante la lactación y tuvo un $E E_{305}$ de sólo $3.3 \mathrm{~kg}$. El número de lactaciones y la estación al parto tuvieron un efecto significativo sobre la mayoria de los parámetros básicos y derivados de los modelos de curva de lactación, mientras que el efecto del FEN sólo afectó el parámetro $\beta_{0}$ del modelo exponencial negativo y a la producción máxima en el modelo gamma incompleto. Los tres factores mencionados tuvieron efecto significativo sobre la producción total pronosticada por campaña de 305 dáas.

Palabras clave: curva de lactación, modelo matemático, vaca lechera, producción de leche

\section{INTROIUCCION}

El uso de modelos matemáticos, tanto mecanisticos como empíricos, ha permitido describir curvas de lactación en los diferentes sistemas de producción lechera. Sin embargo, no todos los modelos se adecúan a una curva de lactación tipica, con fases secuenciales de producción ascendente, máxima y descendentc. Un modelo adecuado seria aquel que permita predecir la producción máxima y el lapso de tiempo para que ella ocurra, la capacidad de mantener la producción durante la fase descendente (persistencia) y la producción total de una campaña estándar de 305 dias. Asimismo, los parámetros de un modelo adecuado de la curva de lactación deben reflejar la influencia de factores genéticos o las interrelaciones fisiológicas, reproductivas y medioambientales $y$, de esta manera, predecir el comportamiento productivo individual o grupal del hato; aun en situaciones particulares como la estación de verano o el fenómeno El Niño de los años $1997 \times 1998$.
Dentro de los modelos matemáticos empíricos que permiten describir una curva de lactación están los exponenciales negati$\operatorname{vos}\left(\hat{y}=\beta_{0} e^{-\beta_{1} x}\right)$, gamma incompletos $\left(\hat{y}=\beta_{0} x^{\beta_{1}} e^{-\beta_{2} x}\right)$ y los del tipo polinomial $\left(y=\beta_{0}+\beta_{1} x+\ldots+\beta_{n} x^{n}\right)$, los cuales permiten estimar el promedio de la producción de leche $(\hat{y})$ en un tiempo dado $(x)$. Cada uno de estos modelos tiene ventajas y desventajas propias. Los modelos exponenciales negativos fueron las primeras aproximaciones empiricas a la curva de lactación y su énfasis descriptivo radica en la persistencia. Los modelos gamma incompletos son los más usados para describir la curva de lactación de bovinos y otras especies. Los modelos polinomiales son simplificaciones lineales de la curva de lactación.

Se considera que el ajuste de modelos matemáticos a las curvas de lactación, as como la estimación del efecto de factores no genéticos sobre los parámetros producidos por los modelos debe proporcionar criterios adecuados, tanto en la toma de decisiones para hacer más eficientes los sistemas de produc- 
ción de leche como para el diseño experimental en trabajos de investigación. En este contexto, los objetivos del presente estudio fueron: (a) ajustar comparativamente los modelos exponencial negativo, gamma incompleto y polinomial de quinto orden a curvas de lactación de vacas Holstein de la cuenca lechera de Lima y (b) determinar el efecto de factores no genéticos como número de lactaciones, estación de parto y condiciones del fenómeno El Niño (FEN) sobre los parámetros estimados a partir de los datos de producción de leche registrados entre los años 1997 a 2001.

\section{Materiales y Métodos}

Los datos de producción de leche fueron proporcionados por cinco establos de la cuenca de Lima, dos ubicados en la zona norte, dos en la zona sur y uno en la zona central. Geográficamente, la ubicación de los establos abarca los paralelos $11^{\circ} 07^{\prime} \mathrm{S}$ a $12^{\circ} 16$ ' S, con una altitud promedio de 137 $\mathrm{msnm}$. Las condiciones particulares de manejo en los establos incluidos en el estudio son de un sistema estabulado intensivo, con alimentación en base a concentrados y forrajes, aplicación de ordeño mecánico dos veces al día, y con manejo reproductivo mediante inseminación artificial.

\section{Procesamiento y depuración de datos}

Se contó con un total de 12,479 registros mensuales provenientes de 1,022 lactaciones correspondientes al periodo 1997 2001. Los datos almacenados en la matriz fueron sometidos a una depuración inicial donde se excluyeron aquellos registros que procedían de lactaciones inducidas o con uso de somatrotropina, de animales que parieron antes de enero de 1997 ó después de diciembre de 2001, de lactaciones cuya fecha del primer registro era posterior a los 31 días del parto, las lactaciones cuya duración era menor a 150 ó mayor a 550 días, de vacas cuyo registro no presentaba fecha de nacimiento, de parto o de colección de la observación, de vacas con más de 7 lactaciones o de lactaciones cuyos registros hubieran sido tomados con intervalos superiores a los 45 días entre registros. En consecuencia, para este estudio se emplearon un total de 9,979 registros provenientes de 833 lactaciones.

Con el propósito de determinar el efecto de factores no genéticos sobre los parámetros estimados que definen la curva de lactación, las lactaciones resultantes del proceso de depuración, fueron reagrupadas por número de lactaciones con las categorías Lactación 1, 2, 3 y 4+ (para las lactaciones provenientes del primer, segundo, tercer y cuarto o más partos), Estación de parto (con las categorías verano, otoño, invierno y primavera, correspondiente a aquellas lactaciones cuya fecha de parto estuvieran entre los meses de diciembre a febrero, de marzo a mayo, de junio a agosto y de setiembre a noviembre, respectivamente) y Fenómeno El Niño (con categorías de "con verano El Niño" y de "verano normal", correspondientes a lactaciones cuya fecha de parto fuese de enero a marzo de 1998 para el primer caso, y de enero a marzo de 1997, 1999, 2000 y 2001 para el segundo caso).

\section{Ajuste comparativo de los modelos}

Tres modelos matemáticos (exponencial negativo, gamma incompleto y polinomial de quinto orden) fueron ajustados comparativamente a las 833 lactaciones provenientes de una primera depuración. Se usaron dos aproximaciones al ajuste comparativo: una global o a través de lactaciones, y otro individual o dentro de lactaciones. La primera consistió en ajustar los modelos al conjunto de los 9,979 registros como si fueran una sola lactación, sin considerar número de lactación, época de parto ni procedencia. La segunda consistió en ajustar individualmente las 833 lactaciones y comparar los modelos en términos de los promedios y desviaciones estándar de los parámetros. 
El modelo exponencial negativo (Brody et $a t$. 1923, citado por Scherchand et at., 1995; Landete-Castillejos y Gallego, 2000; Lucas, 1960) tiene la forma $\hat{y}=\beta_{0} e^{-\beta_{1} x}$ y ha sido utilizado principalmente para describir la fase descendente de la lactación. Con este modelo se estimó la producción ajustada a 305 dias mediante $\hat{y}_{305}=\beta_{0} e^{-\beta_{2}(305)}$ y la producción total estándar (305 d) de cada lactación a través de

$$
\int_{0}^{305} \beta_{0} e^{-\beta_{1} x} d x=\beta_{0}\left(\frac{1}{\beta_{1}}-\frac{e^{-305 \beta_{1}}}{\beta_{1}}\right)
$$

El modelo garnma incompleto, propuesto por Wood en 1967 (citado por Wood, 1970) fue utilizado en su forma no lineal: $\left(\hat{y}=\beta_{0} x^{\beta, z-\beta, x}\right)$. Este modelo ha sido extensivamente usado para describir curvas de lactación de bovinos (León Velarde et al., 1995; Scherchand et al., 1995; Vargas et al., 2000), de ovinos (Pulina et al, 2001; A folayan et al, 2002), de cabras (Akpa et al., 2001) y de camélidos sudamericanos (Quiroz, 1992). El modelo permitió estimar la producción en el pico de lactación $(X p)$ mediante $\beta_{0}\left(\beta_{1} / \beta_{2}\right)^{\beta_{1}} \mathrm{e}^{-\beta_{1}}$; el tiempo en el cual se alcanza el pico de producción $(D P)$, a través de $\beta_{1} / \beta_{2}$ y la persistencia $(s)$ mediante $\left(\beta_{t}+1\right) \ln \beta_{z}$. Análogamente al modelo anterior, la producción estimada a 305 días fue calculada a partir de $\beta_{0}(305)^{\beta_{i}} e^{-\beta_{*}(305)}$ y la producción total estándar ( 305 d) para cada lactación mediante la expresión

$$
\int_{0}^{305} \beta_{0} x^{\beta_{k}} e^{-\beta_{2} x} d x
$$

El tercer modelo fue el polinomial de quinto orden, utilizado por Arango et al. (2000) y tiene la forma $\hat{y}=\beta_{0}+\beta_{1} \mathrm{x}+\beta_{2} \mathrm{x}^{2}+\beta_{3} \mathrm{x}^{3}+$ $\beta_{4} x^{4}+\beta_{5} x^{5}$. La producción de leche al dia 305 fue obtenida mediante $\beta_{0}+\beta_{1}(305)+$ $\beta_{2}(305)^{2}+\beta_{3}(305)^{3}+\beta_{4}(305)^{4}+\beta_{5}(305)^{5}$ y la producción total de la lactación estándar mediante la siguiente forma

$$
\int_{0}^{305} \beta_{0}+\beta_{1} x+\beta_{2} x^{2}+\beta_{3} x^{3}+\beta_{4} x^{4}+\beta_{5} x^{5} d x
$$

Los parámetros $\beta_{i}$ de los modelos exponencial negativo y gamma incompleto fueron estimados mediante regresion no lineal usando el procedimiento PROC NLIN del SAS/STAT ${ }^{\text {MM }}$ (SAS Institute Inc., 1985) con el proceso iterativo Marquardt (Draper y Smith, 1981) y para el modelo polinomial de quinto orden mediante regresión múltiple usando el procedimiento PROC REG de SAS/ STAT. Los parámetros derivados (producción máxima al pico, tiempo a la producción máxima al pico y la persistencia) del modelo gamma incompleto y el referido a la producción estimada a 305 dias de los tres modelos fueron calculados con la ayuda de una hoja de cálculo electrónica. Las estimaciones para la producción total de la lactación estándar $\left(Y_{30 s}\right)$ fueron resueltas analíticamente mediante una hoja electrónica de cálculo para el modelo exponencial negativo. Para los modelos gamma incompleto y polinomial de quinto orden se empleó un procedimiento de integración numérica con el software QuickMath (c) Copyright 1999-2003 QuickMath).

Para fines de comparación entre los tres modelos aplicados, en cuanto a su bondad de ajuste, se consideraron las siguientes estimaciones:

(a) El coeficiente de determinación múltiple ajustado $\left(R^{2}{ }_{a f}\right)$ :

$$
R_{y}^{2}=1-\left(1-R^{2}\right)\left(\frac{n-1}{n-p}\right)
$$

donde $\left(R_{a f}^{2}\right)$ es el coeficiente de determinación múltiple del modelo pero con un ajuste por el número de observaciones $(n)$ y por el número de parámetros $(p)$ involucrados en el modelo y $R^{2}=1-(S C R /$ $S C T$, donde SCR es la suma de cuadrados residual y $S C T$ es la suma de cuadrados total del modelo aplicado (Draper y Smith, 1981; Vargas et al, 2000).

(b) El errơ estándar residual (EER).

$$
E E R=\sqrt{\frac{S C R}{n-p}}
$$

(c) El error estăndar de la predicción individual de la producción en el dia $305\left(E E_{305}\right)$. 


\section{Efecto de factores no genéticos sobre la curva de lactación}

El efecto de los factores no genéticos fue estimado mediante un conjunto de modelos lineales generales:

$Y_{i j k}=L A C_{i}+S T A_{j}+e_{i j k}$

$Y_{i j k}=E S T_{i}+S T A_{j}+e_{i j k}$

$Y_{i j k}=F E N_{i}+S T A_{j}+e_{i j k}$,

donde:

$L A C_{i}=$ efecto del número de lactaciones $(i=1,2,3,4+)$

$E S T_{i}=$ efecto de la estación de parto $(i=$ verano, otoño, invierno, y primavera)

$F E N_{i}=$ efecto de El Niño $(i=$ verano El

Niño, y verano normal)

$S T A_{j}=$ efecto de establo $(j=1,2, \ldots, 5)$

$e_{i j k}=$ residuo aleatorio

Para todos los casos, tanto el efecto de establo como del error fueron considerados como factores aleatorios. La solución estadística del efecto de cada factor fue estimada mediante análisis de varianza usando el procedimiento PROC GLM del paquete estadístico SAS/STAT ${ }^{\mathrm{TM}}$ (SAS Institute Inc., 1985). La comparación entre las diferentes categorías para cada uno de los factores fue realizada mediante la prueba de comparación múltiple de medias de Duncan (Snedecor y Cochran, 1967).

\section{Información meteorológica}

Las condiciones meteorológicas de Lima durante el periodo de evaluación estuvieron caracterizadas por una precipitación pluvial anual escasa (promedio $2.4 \mathrm{~mm}$ ), con una temperatura entre 14.5 y $29^{\circ} \mathrm{C}$ y una humedad relativa de hasta $98 \%$. El periodo marzo 1997 a febrero 1998 fue oficialmente declarado bajo efecto del fenómeno El Niño (SENAMHI, 1999). Para medir el posible efecto del estrés calórico sobre el comportamiento de las curvas de lactación, se estimó el índice temperatura-humedad (ITH) de acuerdo al procedimiento de Huber (1996) y Ravagnolo et al. (2000).

\section{Resultados y Discusión}

El análisis preliminar de los datos de cada lactación mostró algunas anomalías comunes en este tipo de registros. Así, 0.36\% de las lactaciones no convergieron a la iteración de Marquardt al momento de efectuar los ajustes empleando el modelo exponencial negativo y/o gamma incompleto. Asímismo, contradictoriamente a lo esperado, existieron lactaciones que generaron estimados negativos de $\beta_{1}(6.12 \%)$ y de $\beta_{2}(3.12 \%)$ en el modelo gamma incompleto. Dichas lactaciones fueron excluidas del análisis para los ajustes de los tres modelos aquí empleados, de acuerdo a lo recomendado en la literatura (Ali et al., 1996; Tekerli et al., 2000). Análogamente, para el ajuste del modelo polinomial de quinto orden sólo se consideraron lactaciones que tuvieran uno o más grados de libertad, excluyéndose por lo tanto, aquellas lactaciones con menos de siete registros. Por consiguiente, se llegaron a utilizar 8,763 registros correspondientes a 770 lactaciones para los ajustes exponencial negativo y gamma incompleto y de 7,828 registros correspondientes a 647 lactaciones para el ajuste del modelo polinomial de quinto orden.

\section{Ajuste comparativo de los modelos}

Los resultados de la comparación a través de lactaciones se muestran en el Cuadro 1. El modelo polinomial de quinto orden obtuvo el mayor $R^{2}{ }_{a j}$, lo cual se ve reflejado en los menores valores del $E E R$ y del $E E_{305}$. En contraste, la menor bondad de ajuste se presentó con el modelo exponencial negativo, explicable en parte porque este modelo no es capaz de predecir el pico de máxima producción de leche que se presenta durante la lactación temprana.

Los bajos valores de $R_{a j}^{2}$ obtenidos a través de lactaciones, son explicables por la gran dispersión y amplitud de puntos que presentan los registros individuales alrededor de la curva de lactación promedio o ajustada para cada modelo (Fig. 1). Además, se debe con- 
Cuadro 1. Estimados de la bondad de ajuste de tres modelos a través de lactaciones y dentro de lactaciones según el coeficiente de determinación múltiple ajustado $\left(\mathrm{R}_{\mathrm{aj}}^{2}\right)$, el error estándar residual (EER) y el error estándar de la predicción de la producción en el dia $305\left(\mathrm{EE}_{305}\right)$

\begin{tabular}{lllll}
\hline \multicolumn{1}{c}{ Modelo } & No & $\mathrm{R}_{\mathrm{aj}}^{2}$ & EER & EE \\
\hline A través de lactaciones & & & & \\
\hline Exponencial negativo & 8,763 & 0.3122 & 7.129 & 7.131 \\
Gamma incompleto & 8,763 & 0.3811 & 6.763 & 6.764 \\
Polinomial quinto & 7,828 & 0.4056 & 6.595 & 6.598 \\
\hline Dentro de lactaciones & & & & \\
\hline Exponencial negativo & 770 & 0.4880 & 4.4497 & 4.9683 \\
Gamma incompleto & 770 & 0.7251 & 2.9822 & 3.2803 \\
Polinomial quinto & 647 & 0.8359 & 2.1090 & 8.7044 \\
\hline
\end{tabular}

siderar que, por ser un hecho biológico, ha de existir variabilidad entre individuos genéticamente idénticos. Esta variabilidad es acentuada debido a que cada registro individual de producción refleja, no sólo el efecto del día en lactación, sino también el efecto de factores tales como manejo individual de los animales, número de lactaciones, día del año, año y estación de parto y manejo del hato.

Aparentemente, la capacidad predictiva del ajuste de los modelos a través de lactaciones es limitada; sin embargo, los parámetros obtenidos para el modelo gamma incompleto están dentro del rango obtenido para vacas Holstein en diversas latitudes (Wood, 1970; Arango et al., 2000; Farhangfar et al., 2000; Tekerli et al., 2000; Ben Gara et al., 2002). Una particularidad aparente del modelo polinomial de quinto orden es que al final de la lactación presentan una curva ascendente de respuesta (Fig. 1c). Esta respuesta no es plausible biológicamente y preliminarmente podemos considerarla como un artefacto del modelo.

Al hacer el análisîs dentro de lactaciones se observa que el ajuste de los modelos mejora notablemente. Si se compara a través de lactaciones y dentro de lactaciones se encuentran dos aspectos que pueden ser resaltados:
En primer lugar, el ajuste dentro de lactaciones elevó el $R^{2}{ }_{a j}$ de los modelos exponencial negativo, gamma incompleto y polinomial quinto en aproximadamente 56,90 y $106 \%$, respectivamente; y en forma concurrente, el $E E R$ y el $E E_{30.5}$ se redujeron significativamente. En segundo lugar, el $E E_{305}$ del modelo polinomial quinto es mucho mayor que el de los otros dos modelos, y este mismo se incrementó en lugar de reducirse al cambiar de un ajuste al otro. Estos últimos resultados sugieren que sí bien el modelo polinomial quinto tiene una superior capacidad de ajuste a lo largo de toda la lactación, el mismo tiene dificultades para predecir la producción en la fase tardía de la lactación, dejando como mejor opción al modelo gamma íncompleto, el cual puede aun ser mejorado con la inclusión de un efecto para dias del año en las lactaciones individuales (Grossman et al., 1986).

\section{Efecto de los factores no genéticos so- bre la curva de lactación}

\section{Nümero de lactaciones}

Los resultados indican que el número de lactaciones tuvo un efecto significativo en la mayoria de los estimados de los parámetros básicos y derivados de los modelos de curva de lactación. Asi, el Cuadro 2 muestra la in- 


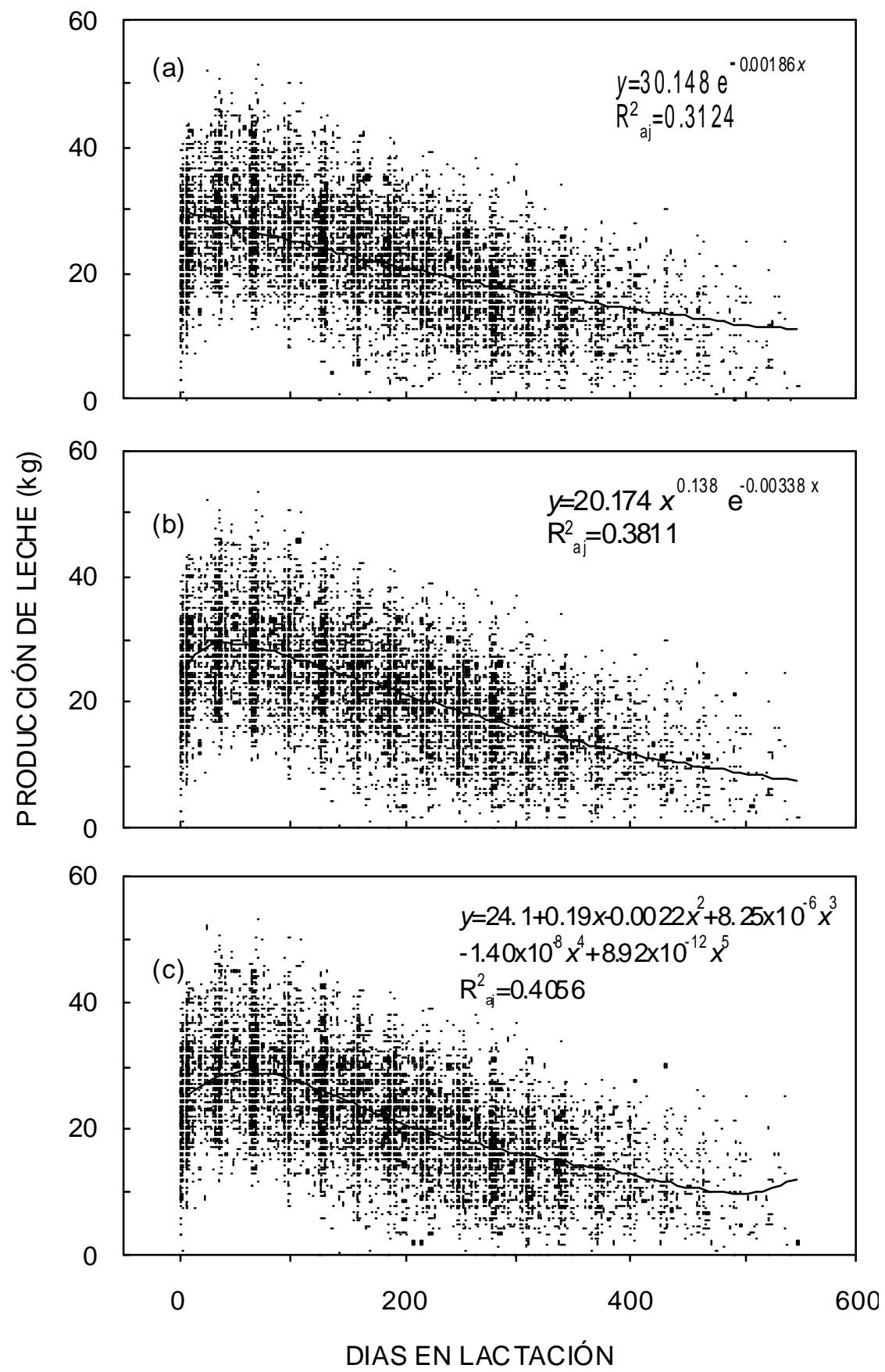

Figura 1. Diagramas de dispersión de puntos de la curva de lactación ajustadas por los modelos exponencial negativo (a), gamma incompleto (b), y polinomial quinto (c) para el total de registros de producción de la cuenca lechera de Lima (1997-2001) 
fluencia del número de lactaciones sobre los valores de los parámetros. Hasta la tercera lactación, el número de lactaciones tuvo una influencia positiva sobre los estimados de los parámetros, asi como sobre los pronósticos de la producción total a los 305 dias de los tres modelos, y más específicamente para el gamma incompleto (el más descriptivo). Después de esta lactación, hubo tendencia a la estabilización o a un ligero decrecimiento, contrariamente a lo informado por Horman y Wattiaux (1996), quienes señalan que la producción de leche tiende a maximizarse recién en la cuarta o quinta lactación.

Los resultados obtenidos confirmarian lo que ha sido observado en otras latitudes: a un mayor número de lactaciones, las vacas tienden a incrementar sus producciones máximas al pico de lactación ( $Y p)$, el tiempo $(D p)$ en llegar a ese pico tiende a ser menor y las vacas tienden a ser menos persistentes (Wood, 1970; Ali et al., 1996; Tekerli et al., 2000; Ben Gara et al., 2001). Esta tendencia estaría asociada con un mayor desarrollo de la glándula mamaria a través de las sucesivas lactaciones en vacas multíparas $y$, por consiguiente, con una mayor tasa de proliferación de las células secretorias mamarias antes del parto y durante la lactación temprana (Akers, 2000). Además, es probable la existencia de un tejido secretor del final de la lactación previa, el cual estaría disponible con un efecto acumulativo para la siguiente lactación (Pollot, 2000). Asimismo, la mayor persistencia mostrada por las vacas en su primera lactación podria ser debida a que el proceso de maduración de la glándula mamaria estaría aún en progreso por tratarse de vacas jóvenes, el cual estaría generando que la tasa de disminución del rendimiento sea más lenta durante la lactación tardía (Catillo et al., 2002).

\section{Estación de parto}

Los resultados del análisis de variancia sugieren un posible efecto estacional sobre los parámetros de las respectivas curvas de lactación. El parámetro $\boldsymbol{\beta}_{0}$ de los modelos de ajuste exponencial negativo y gamma in- completo para vacas que parieron en el verano de 1997 al 2001 fue significativamente menor en comparación con aquellas vacas que parieron en invierno (Cuadro 2). Similar condición se produce con el parámetro $\beta_{1}$ de la función gamma incompleta (fase ascendente), presumiblemente como consecuencia del estrés de calor que pudieran haber tenido en el verano. En dichos veranos se estimó que el ITH promedio para los años en estudio fue 76 , valor superior a 72 el cual es considerado como el umbral de confort para el animal (Ravagnolo et al., 2000). El ITH promedio para el invierno fue de 66 , lo cual sugiere que los menores valores de los parámetros $\beta_{0}$ y $\beta$, para el modelo gamma incompleto en el verano estarían asociados mayormente con una reducción en el consumo total de materia seca por efecto del estrés calórico que sufre el animal (West, 2003) y en consecuencia con el deterioro de su balance energético y su homeostasis.

En relación a la producción máxima $\left(Y_{p}\right)$, aquellos animales que parieron en otoño, invierno o primavera huvieron producciones pico significativamente más elevadas que aquellas vacas que parieron durante el verano (Cuadro 2). Hecho que podría explicarse debido a que el estrés calórico soportado por el animal que parió durante el verano (ITH de 76) no le permitió expresar todo su real potencial genético de producción de leche, mientras que sí pudieron hacerlo aquellos animales que parieron en las estaciones cuyos ITH fueron inferiores a 72 (ITH de 71, 66 y 70 para el otoño, invierno y primavera, respectivamente). Lo anteriormente expuesto se reflejó en que aquellas vacas que parieron en otoño o invierno presentaron las mayores producciones totales de leche.

Estos resultados confirman las observaciones de López (1966) en la cuenca lechera de Lima, quien encontró la más baja producción total en aquellas vacas que parieron en la primavera. También son análogos a los observadas en otras latitudes como Arabia Saudita, $24^{\circ} \mathrm{N}$ (Ali et al., 1996), Portugal, $40^{\circ} \mathrm{N}$ (Silvestre et $a l ., 1997$ ) y Túnez, $32^{\circ} \mathrm{N}$ (Ben Gara et al., 2002). 


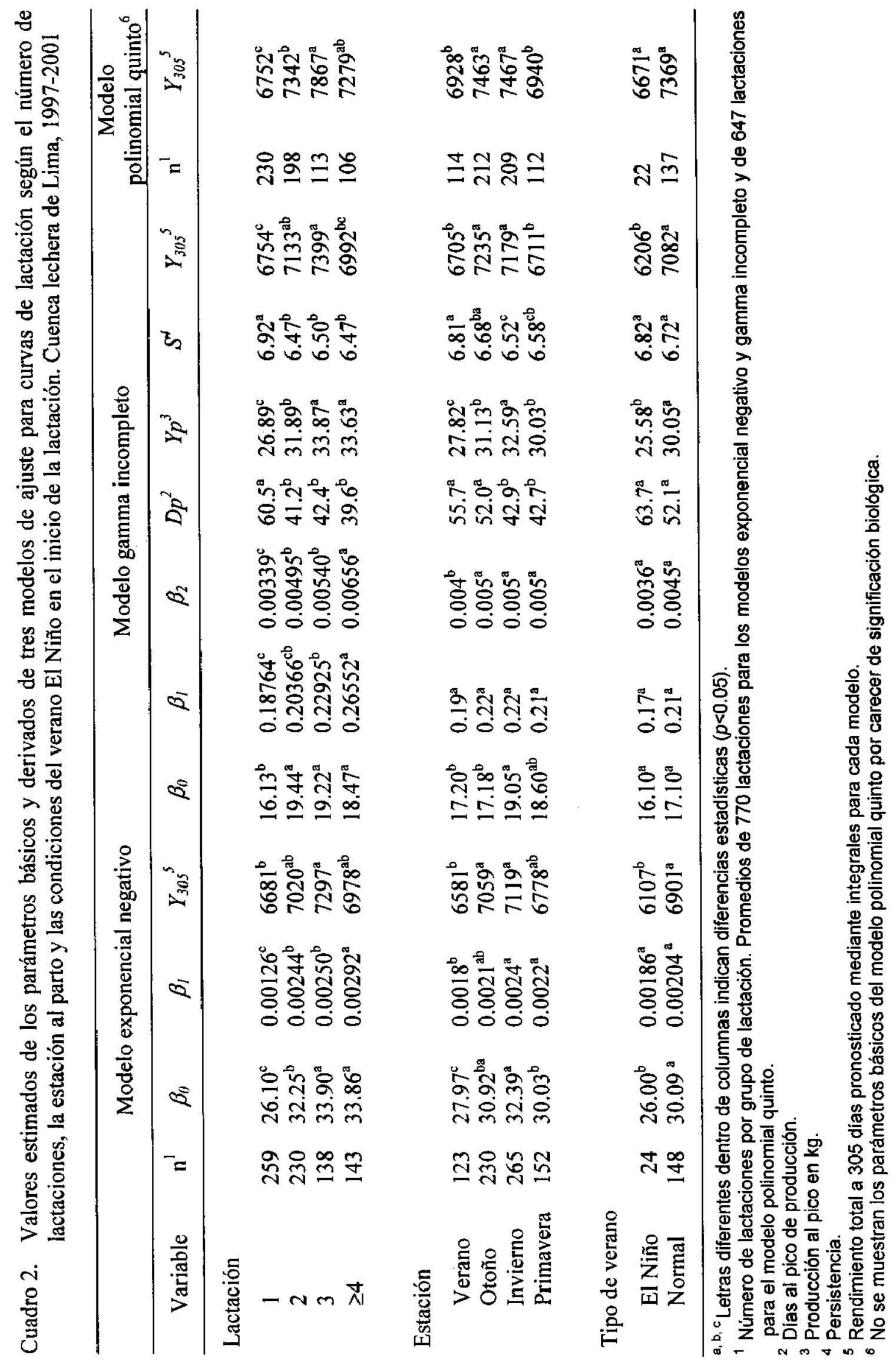




\section{Fenómeno E1 Niño 1998}

El análisis de variancia mostró que el FEN afectó significativamente al parámetro $\beta_{0}$ (producción inicial de la lactancia) del modelo exponencial negativo $\mathrm{y}$ a la producción máxima (Yp) estimada por el modelo gamma incompleto (Cuadro 2). Esta situación estaria asociada a los más altos valores promedio de ITH durante dicho periodo (ITH de 79) en comparación con los promedios presentados en los veranos considerados como normales (ITH de 75), lo cual trajo como consecuencia un mayor estrés calórico en los animales del primer grupo, con efectos adversos sobre su consumo de alimento y consecuente deterioro de su balance energético y de su homeostasis. Los efectos del FEN, tanto sobre el parámetro $\beta_{0}$ del modelo exponencial negativo como la producción máxima al pico $(Y p)$ del modelo gamma incompleto se reflejarian más directamente en los promedios de producción total a 305 días $\left(Y_{30}\right)$, debido a que los menores valores de $\beta_{0}$ y de $Y p$ fueron suficientes para producir mermas significativas en la producción total pronosticada para una lactación estándar (Cuadro 2). Esto sería concurrente con la afirmación de Whittemore (1980) quien considera a lp como el parámetro mejor asociado con la producción total, conjetura que podría ser considerada para estudios posteriores.

\section{Conchenones}

- El modelo gamma incompleto es la opción recomendable para utilizar en la cuenca de Lima, el cual es capaz de explicar el $72 \%$ de la variación en producción dentro de una lactación y de tener sólo $3.3 \mathrm{~kg}$ de error estándar para pronósticos de producción de leche en el día 305.

- El ajuste dentro de lactaciones mejora sensiblemente la capacidad de producción de los modelos a través de un incremento del coeficiente de determinación y una reducción en los errores estandar, tanto del modelo como de la predicción de la producción a 305 días.

- El número de lactaciones, hasta la tercera lactancia, tuvieron una relación directa con los estimados de los parámetros que reflejan la producción inicial, la tasa de ascenso de la producción, la producción máxima al pico, y el rendimiento de la producción a 305 dias; pero una relación inversa con los dias al pico y la persistencia. A partir de la cuarta lactancia los parámetros tienden a estabilizarse y/o a disminuir.

- Los partos en verano, estación de estrés calórico para el animal (ITH $>72$ ), tuvieron un efecto negativo sobre los parámetros que reflejan la producción inicial, la tasa de descenso, la producción máxima al pico y en el rendimiento total de la lactancia a 305 dias. Estas dos últimas caracteristicas se agudizan cuando esta estación coincide con un evento de E1 Niñon.

\section{LMFRATURA CTADA}

1. Afolayan, R.A.; B.Y. Abubakar; O.A. Osinowo, N.I. Dim. 2002. Lactation and function of curve parameters in Yankasa sheep. Asian-Aust. J. Anim. Sci. 15:890 894.

2. Akers, R.M. 2000. Selection for milk production from a lactation biology viewpoint. J. Dairy Sci. 83: 151-1158.

3. Akpa, GN. E. O. Asiribo; O. Oni; J.R. Alawa, 2001. The influence of nongenetic factors on the shape of lactation curves in Red Sokoto goats, Anim. Sci. 72:233-239.

4. Ali, A.K.A.; R.S. Al-Jumaah; E. Hayes. 1996. Lactation curve of Holstein-Friesian cows in the Kingdom of Saudi Arabia. Asian-Aus. J. Anim. Sci. 9: 439,447 . 
5. Arango, J.P.; B. Rivera; J.C. Granobles. 2000. Elaboración y validación de modelos de estimación de producción lechera en sistemas especializados. Trabajo de grado, Facultad de Ciencias Agropecuarias, Universidad de Caldas. En: Recopilación y Sistematización de los resultados de investigación de la Facultad de Ciencias Agropecuarias de la Universidad de Caldas 1950-2000. (CD ROM). Colombia.

6. Ben Gara, A.; B. Rekik; M. Ben Hamouda; M. Guellouz. 2002. Recording system and quality of dairy cattle production data in Tunisia. Proc. 2002 Interbull Meeting. p 196-199. Interlaken, Suiza.

7. Catillo, G.; N.P. Macciotta; A. Carretta; A. Cappio-Borlino. 2002. Effects of age and calving season on lactation curves of milk production traits in Italian water buffaloes. J. Dairy Sci. 85: 1298-1306.

8. Draper, N.R.; H. Smith. 1981. Applied regression analysis. $2^{\text {d }}$ edition. John Wiley \& Sons. NY. 709 p.

9. Farhangfar, H.; P. Rowlinson; M.B. Willis. 2000. Estimation of lactation curve parameters for Iranian Holstein dairy cows using non linear models. Poster Session 109. Proc. Brit. Soc. Anim. Sci. Meeting 2000. Scarborough, UK. Disponible en: http://www.bsas.org.uk/meetings/ annlproc/PDF2000/109.pdf

10. Grossman, M.; A.L. Kuck; A.H. Norton. 1986. Lactation curves of purebred and crossbred dairy cattle. J. Dairy Sci. 195-203.

11. Homan, E.J.; M.A. Wattiaux. 1996. Guía técnica lechera: lactancia y ordeño. Instituto Babcock. Universidad de Wisconsin-Madison. USA. $101 \mathrm{p}$.

12. Huben J.T. 1996. Mejora del estrés por el calor en el ganado vacuno lechero. En: Avances de la ciencia de la produccion lechera. Cap. 11. C.J.C. Phillip (ed). p 217-219. Ed. Acribia. España.

13. Landete-Castillejos, T.; L. Gallego. 2000. The ability of mathematical models to describe the shape of lactation curves. J. Anim. Sci. 78: 3010-3013.
14. Leon-Velarde, C.U.; I. McMillan; R.D. Gentry; J.W. Wilton. 1995. Models for estimating typical lactation curves in dairy cattle. J. Anim. Breed. Genet. 112: 333-340.

15. López, M.F. 1966. Variaciones estacionales en la producción de leche y el porcentaje de grasa en la cuenca lechera de Lima y alrededores. Tesis Ing. Zootecnista. UNALM. Lima. 129 p.

16. Lucas, H.L. 1960. Design and analysis of feeding experiments with milking dairy cattle. North Carolina State University. Mimeo Series 18. Raleigh, NC.

17. Pollot, G.E. 2000. A biological approach to lactation curve analysis for milk yield. J. Dairy Sci. 83: 2448-2458.

18. Pulina, G.; A. Cappio-Borlino; N. Macciotta; C. Di Mauro; A. Nudda. 2001. Empirical and mechanistic mathematical models of temporal evolution of milk production in ruminants. Riv. Biol. 94: 331-344.

19. Quiroz, R. 1992. Apuntes sobre modelos matemáticos en problemas ganaderos. En: Simulación de sistemas pecuarios. Red de Investigación en Sistemas de Producción Animal en Latinoamérica. M.E. Ruiz (ed). p 157-187. RISPAL/ IICA. San José, Costa Rica.

20. Ravagnolo, O.; I. Mistral; G. Hoogenboom. 2000. Genetic component of heat stress in dairy cattle: development of heat index function. J. Dairy Sci. 83: 2120-2125.

21. SAS Institute Inc. 1985. SAS/STAT ${ }^{\mathrm{tm}}$ Guide for personal computers Version 6. Cary, NC., USA. 379 p.

22. Scherchand, L.; R.W. McNew; D.W. Kelloqq; Z.B. Johnson. 1995. Selection of a mathematical model to generate lactation curves using daily milk yields of Holstein cows. J. Dairy Sci. 78: 25072513.

23. SENAMHI. 1999. Fenómeno El Niño 1997/98. Lima, Perú. 65 p.

24. Silvestre, A.; F. Petim-Baptista; J. Colaço. 1997. Lactation curve seasonality. Rev. Port. Zoot. 5(2). Dis- 
ponible en: http://home.utad.pt/apez/ Publicacoes/revista/ano-y-2/art-02.htm

25. Snedecor, GW.; W.G Cochran. 1967. Statistical methods. $6^{\text {th }}$ ed. p 274 . Iowa State University Press. Ames, IA. USA.

26. Tekerli, M.; Z. Akinci; L. Dogan; A. Akcan. 2000. Factors affecting the shape of lactation curves of Holstein cows from the Balikesir Province of Turkey. J. Dairy Sci. 83: 1381-1386.
27. Vargas, B.; W.J. Koops; M. Herrero; J.A. Van Arendonk. 2000. Modeling extended lactations of dairy cows. J. Dairy Sci. 83: 1371-1380.

28. West, J.W. 2003. Effects of heat-stress on production in dairy cattle. J. Dairy Sci. 86: 2131-2144.

29. Whittemore, C.T. 1980. Lactation of the dairy cow. Longman Group, USA. 94 p. 